\title{
Opioid Receptor PET Reveals the Psychobiologic Correlates of Reward Processing
}

\author{
Mathias Schreckenberger ${ }^{1}$, André Klega ${ }^{1}$, Gerhard Gründer ${ }^{2,3}$, Hans-Georg Buchholz ${ }^{1}$, Armin Scheurich ${ }^{2}$, \\ Ralf Schirrmacher ${ }^{1,4}$, Esther Schirrmacher ${ }^{1,4}$, Christina Müller ${ }^{1}$, Gjermund Henriksen ${ }^{5}$, and Peter Bartenstein ${ }^{1,6}$ \\ ${ }^{I}$ Department of Nuclear Medicine, Johannes Gutenberg-University, Mainz, Germany; ${ }^{2}$ Department of Psychiatry and Psychotherapy, \\ Johannes Gutenberg-University, Mainz, Germany; ${ }^{3}$ Department of Psychiatry and Psychotherapy, Aachen University of Technology, \\ Aachen, Germany; ${ }^{4}$ Department of Neurology and Neurosurgery, McGill University, Montreal, Canada; ${ }^{5}$ Department of Nuclear \\ Medicine, Technical University, Munich, Germany; and ${ }^{6}$ Department of Nuclear Medicine, Ludwig Maximilians-University, \\ Munich, Germany
}

Little is known about the neurobiologic correlates of human personality. On the basis of the key role of the central opioidergic system in addiction and substance abuse, we investigated the relationship between certain personality traits that are supposed to be relevant in addiction and the opioid receptor status in healthy subjects. Methods: We investigated 23 healthy male volunteers who were extensively clinically tested to exclude substance abuse. All of the subjects underwent 1 PET scan with the subtype-nonselective opioidergic radioligand ${ }^{18} \mathrm{~F}$-fluoroethyldiprenorphine under resting conditions without sensory or cognitive stimulation. Subsequently, the subjects were psychologically tested for the personality traits novelty seeking, harm avoidance, reward dependence, and persistence, according to Cloninger's biosocial model of personality. The binding potential (BP) as a parameter of regional cerebral opioid receptor availability was computed by means of the modified Logan plot using the occipital cortex as a reference region. Further imaging data analysis was performed using statistical parametric mapping; after stereotactic normalization, the correlations were calculated between the regional BP and the psychologic scores on a voxel-by-voxel basis. Results: The correlation analysis between personality dimensions and opioid receptor availability showed a significant $(P<0.001)$ positive correlation between the scores of reward dependence and the BP of the bilateral ventral striatum with nucleus accumbens ( $z$ scores, 4.52 and 4.33 , respectively). The additionally performed region-of-interest-based correlation analysis yielded correlation coefficients of $r=0.84$ and $r=0.81$ for the left and right ventral striata, respectively. No further significant correlations were detectable between the other personality dimensions and cerebral opioid receptor binding. Conclusion: In healthy subjects, personality traits, which might be predisposing for addictive behavior, are correlated to the opioidergic neurotransmission in core structures of the human reward system.

Received Jan. 18, 2008; revision accepted Apr. 11, 2008.

For correspondence or reprints contact: Mathias Schreckenberger, Department of Nuclear Medicine, University of Mainz, Langenbeckstrasse 1, D-55101 Mainz, Germany.

E-mail: schreckenberger@nuklear.klinik.uni-mainz.de

COPYRIGHT @ 2008 by the Society of Nuclear Medicine, Inc.
Key Words: opioid receptor; personality; addiction; drug abuse; positron emission tomography

J Nucl Med 2008; 49:1257-1261

DOI: 10.2967/jnumed.108.050849

$\mathbf{T}$ he biologic basis of human personality continues to be one of the most interesting and challenging issues of psychobiologic research. Different approaches have shown that certain personality traits are related to neurobiologic parameters $(1,2)$. The development of dimensional models of personality enables a more appropriate description of the complexity and heterogeneity of personality; these models of personality might also be more closely related to the underlying neurobiologic substrate.

One of the most popular models to describe human personality on a dimensional level is Cloninger's biosocial model of personality $(3,4)$. This model comprises the 4 temperament dimensions novelty seeking, harm avoidance, reward dependence, and persistence and involves automatic, preconceptual responses to perceptual stimuli, most probably reflecting heritable biases in information processing. These dimensions are considered to be stable across time, except for novelty seeking, which seems to be agedependent (5).

Functional imaging using labeled tracers and PET in conjunction with detailed characterization of personality dimensions represents a novel and promising approach for the investigation of the in vivo brain biochemistry of personality. The usefulness of this approach has been documented with radioligands for dopaminergic and serotonergic systems (6). However, little is known about the role of opioidergic neurotransmission for the modulation of human personality, despite the major role that opioidergic neurotransmission plays in the hedonic aspects of reward experience (7). On the basis of this background, it seems challenging to investigate the functional relationship between opioidergic neurotransmission and the level of hereditary personality traits (temperaments) in healthy subjects. 


\section{MATERIALS AND METHODS}

This study was performed in accord with the Helsinki Declaration and was approved by the local ethics committee, the Federal Health Administration, and the radiation protection authorities. All PET investigations were performed at the PET Center of the University of Mainz, Germany.

\section{Subjects}

For this prospective study, 23 healthy male volunteers (age range, 25-54 y) were included. The subjects had no current or previous history of relevant physical illness, no current or past psychiatric disorders, and no family history of a major psychiatric disorder in first-degree relatives, and they were not regularly taking medication. All control subjects received a mental- and physical-state examination including blood analyses. All subjects gave written informed consent.

The subjects underwent a standardized psychologic assessment for the 4 temperament dimensions novelty seeking, harm avoidance, reward dependence, and persistence, according to Cloninger's temperament and character inventory $(3,4)$.

\section{Radiochemistry and Data Acquisition}

${ }^{18} \mathrm{~F}$-fluoroethyl-diprenorphine $\left({ }^{18} \mathrm{~F}-\mathrm{DPN}\right)$ was synthesized by applying the secondary labeling precursor $2-{ }^{18} \mathrm{~F}$-fluoroethyltosylate to 3-O-trityl-6-O-desmethyl-diprenorphine (8). The $2-{ }^{18} \mathrm{~F}$-fluoroethyltosylate was synthesized as described elsewhere (9) and obtained in a diethyl ether solution, which was evaporated in a stream of nitrogen. A solution of $4 \mathrm{mg}$ of 3-O-trityl-6- $O$-desmethyldiprenorphine and $10 \mathrm{mg}$ of sodium hydride in $300 \mu \mathrm{L}$ of $\mathrm{N}, \mathrm{N}$-dimethylformamide was added to the dried $2-{ }^{18} \mathrm{~F}$-fluoroethyltosylate, and the resulting mixture was stirred for $8 \mathrm{~min}$ at $100^{\circ} \mathrm{C}$. Then the reaction mixture was cooled to room temperature, and 600 $\mu \mathrm{L}$ of $\mathrm{HCl}(2 \mathrm{~N})$ were added slowly and stirred for $5 \mathrm{~min}$ at $40^{\circ} \mathrm{C}$. After the mixture was cooled to room temperature, it was diluted with $8 \mathrm{~mL}$ of aqueous ammonia (20\%), stirred for $2 \mathrm{~min}$, and loaded on a Sep-Pak C18 cartridge (Waters Corp.). The product was eluted with $2 \mathrm{~mL}$ of methanol and purified using semipreparative highperformance liquid chromatography (HPLC) ( $\mu$ Bondapak C18 [Waters]: inner diameter, $300 \times 7.8 \mathrm{~mm}$; acetonitrile/0.1N ammonium formate, $55: 45 ; 3 \mathrm{~mL} / \mathrm{min}$, retention time: $14.9 \mathrm{~min}$ ). After diluting the HPLC fraction containing the product with $40 \mathrm{~mL}$ of $0.1 \mathrm{~N}$ ammonium formate, the fraction was loaded on a Sep-Pak C18 cartridge, washed with $10 \mathrm{~mL}$ of water, eluted with $1 \mathrm{~mL}$ of ethanol, and diluted with $9 \mathrm{~mL}$ of physiologic saline solution to yield $1,250-1,950 \mathrm{MBq}$ (radiochemical yield, $19 \% \pm 4 \%$ ) of ${ }^{18} \mathrm{~F}-$ DPN. HPLC analysis (Luna $5 \mu, \mathrm{C} 18(2)$ [Phenomenex]: inner diameter, $250 \times 4.6 \mathrm{~mm}$; methanol/0.1N ammonium formate, 70:30, $1 \mathrm{~mL} / \mathrm{min}$, retention time: $12.2 \mathrm{~min}$ ) showed that the radiochemical purity was greater than $99 \%$, whereas the specific activity (determined via ultraviolet-calibration curve) was between 580 and $820 \mathrm{GBq} / \mathrm{mmol}$.

\section{PET and Data Analysis}

Images were acquired on a whole-body PET scanner (ECAT EXACT; Siemens). The camera had a field of view of $16.2 \mathrm{~cm}$ in 47 planes, with a plane spacing of $3.375 \mathrm{~mm}$, an axial resolution of $6.0-\mathrm{mm}$ full width at half maximum, and an in-plane resolution of $6.0 \mathrm{~mm}$ (resolution at center with scanner in 3-dimensional mode). Data acquisition comprised a series of 30 time frames. The scan duration increased progressively from $20 \mathrm{~s}$ to $10 \mathrm{~min}$, resulting in a total scanning time of $124 \mathrm{~min}$. A 15-min transmis- sion scan using a ${ }^{68} \mathrm{Ge}$ source was performed before each study for subsequent attenuation correction. A mean of $150 \mathrm{MBq}( \pm 30$ $\mathrm{MBq}$ ) of ${ }^{18} \mathrm{~F}-\mathrm{DPN}$ was injected intravenously as a bolus into a cubital vein over approximately $30 \mathrm{~s}$. The specific activity at the time of injection was greater than $0.5 \mathrm{GBq} / \mu \mathrm{mol}$.

Images were reconstructed with filtered backprojection using a ramp filter and a Hanning filter (filter width, $7.3 \mathrm{~mm}$ ). Frameby-frame motion correction was applied by matching cortical isodensity contour points. A mean occipital time-activity curve was generated by drawing regions of interest (ROIs) on 3 subsequent transaxial slices.

Binding potentials (BPs) of volumes of interest were calculated using the noninvasive Logan plot (10), with reference region input, according to the following equation:

$$
\frac{\int_{0}^{t} c_{t} d t}{c_{t}} \cong \frac{V_{d}}{V^{\prime}{ }_{d}} \frac{\int_{0}^{t} c_{r} d t}{c_{t}}+c
$$

where $C_{t}$ is the tissue radioligand activity in the receptorcontaining ROI, $\mathrm{C}_{\mathrm{r}}$ is the tissue radioligand activity of ${ }^{18} \mathrm{~F}-\mathrm{DPN}$ in the reference tissue (occipital cortex), $V_{d}$ is the volume of distribution of the receptor-rich region, and $\mathrm{V}_{\mathrm{d}}^{\prime}$ is the volume of distribution of the reference region (11). BP was calculated from the ratio $\mathrm{V}_{\mathrm{d}} / \mathrm{V}_{\mathrm{d}}^{\prime}$, which was estimated with a nonlinear least-square minimization procedure. The occipital cortex was chosen as a reference region because it is generally considered of very low opioid receptor density (12). BP images had to be normalized stereotactically for voxelwise analysis of opioid receptor availability (13). First, flow-weighted integral images (summed images between 3 and 12 min after injection) were calculated and spatially normalized using statistical parametric mapping and the standard PET template. The images were realigned and stereotactically normalized into the standard anatomic space by means of linear and nonlinear transformation (14). Then, transformation parameters of the spatial normalization were applied to BP images. Subsequently, the normalized BP images were smoothed with a 3-dimensional gaussian filter using a 12-mm full width at half maximum kernel (voxel size, $2 \times 2 \times 2 \mathrm{~mm}$ ).

\section{Statistical Analysis}

For the correlation analysis between personality traits and cerebral opioid receptor availability, the scores of novelty seeking, harm avoidance, reward dependence, and persistence were correlated as an external covariate to the corresponding opioid BP data on a voxelwise basis using a statistical threshold of $P<0.001$ (corrected on a cluster level) and a minimal cluster size of more than 100 voxels. As the image data were stereotactically normalized, the maxima of the activation foci were reported with the respective stereotactic Montreal Neurologic Institute coordinates.

\section{RESULTS}

\section{Personality Assessment}

The personality assessment of the healthy subjects showed mean scores of $21.6 \pm 5.0,10.7 \pm 6.1,15.4 \pm 3.3$, and $5.3 \pm$ 2.0 for novelty seeking, harm avoidance, reward dependence, and persistence, respectively. In comparison with the ageand sex-matched normative data group (10), our volunteers scored slightly above the average in novelty seeking (normative data, $19.0 \pm 5.9$ ), reward dependence (normative 
data, $14.6 \pm 3.5$ ), and persistence (normative data, $4.2 \pm$ 1.8), whereas they scored in the lower average range in harm avoidance (normative data, $14.6 \pm 5.8$ ). No significant differences between the scores of our volunteers and the normative data were identified. The observed differences between our subjects and the normative database were moderate and might be explained by the different social and educational backgrounds of the 2 groups. Whereas the normative data are based on subjects who are representative of the German population, our study subjects were exclusively recruited by participating scientists and students from our departments.

\section{Correlation Opioid Receptor Status and Personality Traits}

The correlation analysis between personality dimensions and opioid receptor availability showed a strong positive correlation between the scores of reward dependence and the BP restricted to the bilateral ventral striata with nucleus accumbens (Fig. 1). The respective stereotactic Montreal Neurologic Institute coordinates of the maximum voxels were $x=-12, y=10$, and $z=-10$ (left) and $x=16, y=$ 26 , and $z=2$ (right); the $z$ scores were 4.52 and 4.33 , respectively. The corrected $P$ values for both clusters were significant at $P<0.001$ (corrected for the entire volume). After small-volume correction, the corrected $P$ values were less than 0.005 at a voxel level within each cluster.

To validate these findings, an ROI-based correlation analysis was subsequently performed using predefined ROIs according to Mawlawi et al. (15) for the ventral striatum. Figure 2 shows the anatomically defined ROI superimposed on significant clusters in the ventral striatum. The corresponding correlation plots are shown in Figure 3; the correlation coefficients were $r=0.81(P<0.0001)$ and $0.84(P<0.0001)$ for the right (Fig. 3A) and left (Fig. 3B) ventral striata, respectively.

In contrast, no correlations passing the given statistical threshold were detectable between opioid receptor binding and the levels of the other personality dimensions.

\section{DISCUSSION}

The findings of this study indicate that opiate receptor availability in the ventral striatum, a core area of the brain reward system, is directly correlated with reward dependence, a personality trait that is thought to reflect a bias in the maintenance of ongoing behavior (4). Recent neurobiologic research has emphasized the difference between the anticipation of reward, which is computed by dopaminergic neurotransmission (16) and is thought to be reflected in the personality trait of novelty seeking (4), and the actual pleasant or hedonic experience of reward, which is blocked by opiate receptor antagonists in the ventral striatum of rodents and in human studies $(7,17)$. Our study confirms this difference and suggests that reward dependence but no other personality trait is correlated with opiate receptor availability in the ventral striatum. For novelty seeking, which is attributed to the dopaminergic system, Suhara et al. (18) detected in healthy volunteers a negative correlation between the scores of novelty seeking and the $\mathrm{BP}$ of dopamine $\mathrm{D}_{2}$ receptors in the right insular cortex. Indications for the involvement of the serotonergic

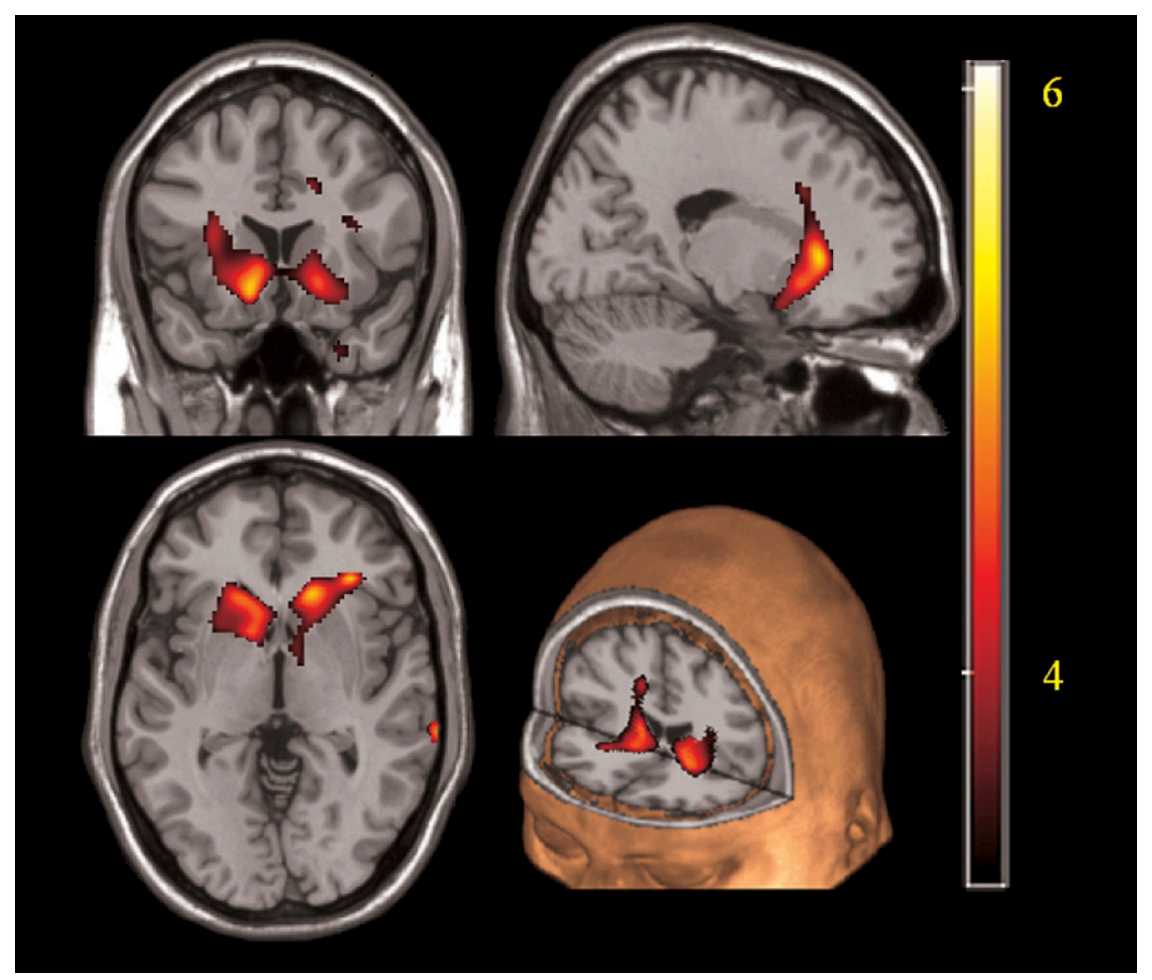

FIGURE 1. Positive correlation (corrected at cluster level at $P<0.001$ ) between score for reward dependence and opioid receptor availability in bilateral ventral striata with caudate nucleus and nucleus accumbens. Color scale shows $t$ levels, with $t=3.55$ corresponding to $P=0.001$. 
FIGURE 2. Predefined anatomic ROIs of ventral striatum superimposed on clusters significant for reward dependence that are shown in Figure 1.

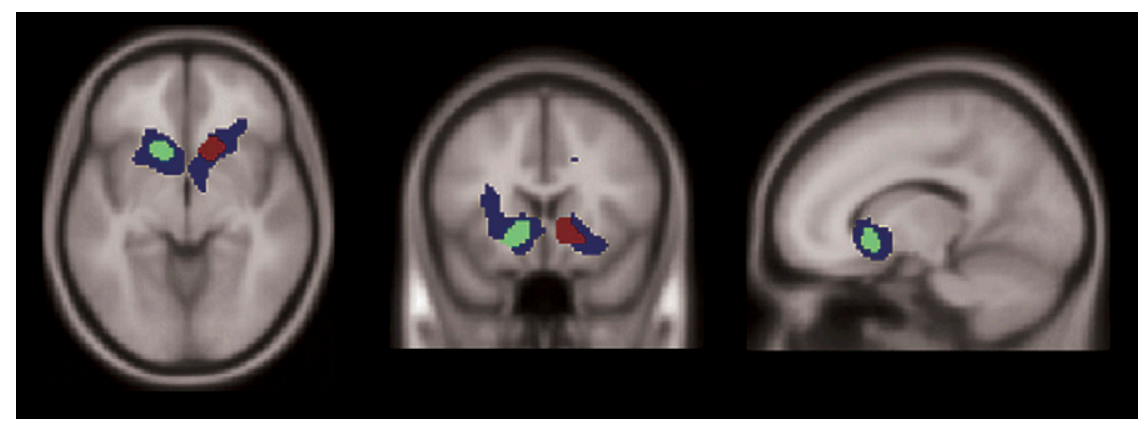

system in certain human personality traits were found by Moresco et al. (6). These authors revealed an inverse correlation between harm avoidance and the binding of ${ }^{18}$ F-fluoroethyl-spiperone to 5-hydroxytryptamine receptor $2 \mathrm{a}$ in the frontal and the left parietal cortex, whereas no serotonergic correlations were found for novelty seeking and reward dependence (6).

Clinically, reward dependence manifests as individual differences in social attachment, dependence on the approval of others, and sentimentality (4). A study in nonhuman primates also suggested that intimate social contact

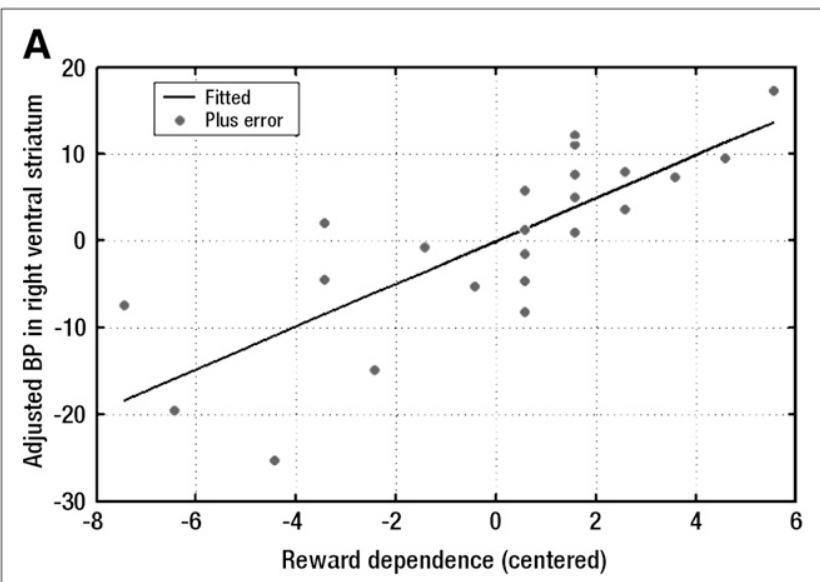

B

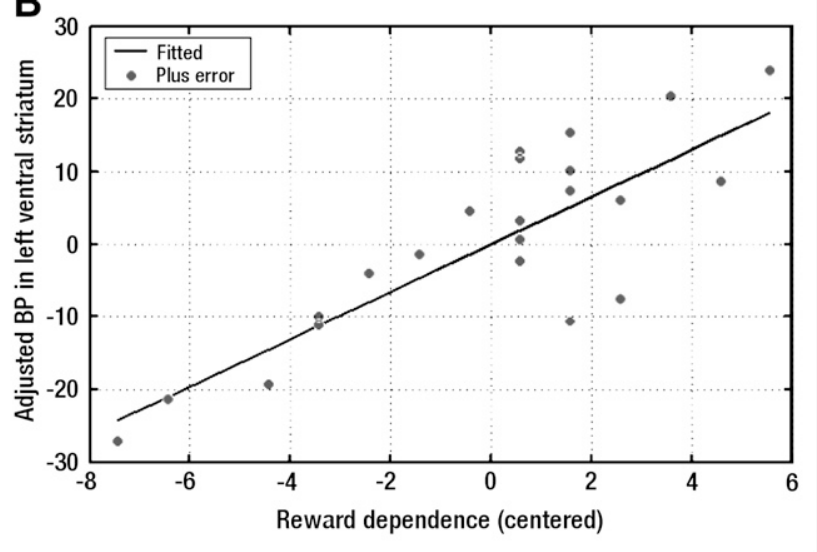

FIGURE 3. Representative plots of extracted binding potential for right $(A)$ and left $(B)$ ventral striata positively correlated to scores of reward dependence (right, $r=0.81$; left, $r=0.84$ ). between a mother and her infant is regulated by the opiate system (19). Our study supports this notion and indicates that opiate receptor availability may mediate reward dependence in social contact behavior via longer-lasting individual differences that can be clinically defined as human personality traits. The clinical relevance of our study is emphasized by findings that link individual differences in opiate receptor availability with the sensory and affective dimensions of pain and with alcohol dependence $(20,21)$. It was shown in a recent PET study that $\mu$-opiate receptor availability in the ventral striatum was increased in abstinent alcoholic patients and was positively correlated with their craving for alcohol as a relevant risk factor for relapse (21).

The increased dopamine release in the ventral striatum (in particular in the nucleus accumbens) due to drug or alcohol administration is mediated and modulated by opioidergic and GABAergic pathways $(22,23)$. Therefore, increased availability of opioid receptors in brain areas that are relevant for positive-reinforcement effects might be important in initiating and maintaining drug or alcohol abuse (24). Thus, our findings suggest that elevated opiate receptors in the ventral striatum could be one biologic link between personality traits and dependence risk.

Some methodic considerations should be addressed. For this study, we assessed the endogenous opioid receptor status in terms of BPs, which were calculated by means of the noninvasive Logan model (10), with the occipital cortex as a reference region. It has been recently shown that the use of noninvasive models seems an appropriate approach for the quantification of ${ }^{11} \mathrm{C}$ - or ${ }^{18} \mathrm{~F}$-labeled diprenorphine $(25,26)$, although the occipital cortex might not fulfill the criteria of an ideal reference region because it may contain a small amount of opioid receptors as could be shown by naloxone-blocking experiments (27). Expecting the specific occipital binding to be minimal, Spilker et al. (26) also used this area as the reference region and found consistent BP values between invasive and noninvasive quantification methods, with the noninvasive BPs showing less interindividual variability. Therefore, the use of the occipital cortex as a reference region might be problematic in those studies using different (activation) conditions, which might influence the specific binding in the occipital cortex depending on the activation paradigm. However, for the present study 
we did not use different conditions, and from our a priori hypothesis, it was not expected that the investigated personality dimensions were associated with opioid receptor binding in the occipital cortex.

\section{CONCLUSION}

The results of this study suggest that opioidergic neurotransmission in the ventral striatum has a role as a biologic modulator between personality traits (reward dependence as revealed by our study) and addiction-related reward and reinforcement processes.

\section{ACKNOWLEDGMENTS}

We thank Andreas Heinz (Department of Psychiatry, Charité Berlin) and Rolf-Detlef Treede (Institute of Physiology, Johannes Gutenberg University Mainz) for their contributions to data analysis and discussion. We also thank Hans-Jürgen Wester (Technical University Munich) for tracer evaluation and help with establishing synthesis in our laboratory. This study was supported by the Deutsche Forschungsgemeinschaft ( $\operatorname{Tr} 236 / 13-3$ ), the Stiftung Rheinland-Pfalz für Innovation, and the MAIFOR research program of the Johannes Gutenberg-University Mainz.

\section{REFERENCES}

1. Costa PT Jr, McCrae RR. Ego development and trait models of personality. Psychol Inq. 1993;4:20-23.

2. Canli T. Functional brain mapping of extraversion and neuroticism: learning from individual differences in emotion processing. J Pers. 2004;72:1105-1132.

3. Cloninger CR. A systematic method for clinical description and classification of personality variants. Arch Gen Psychiatry. 1987;44:573-588.

4. Cloninger CR, Svrakic DM, Przybeck TR. A psychobiological model of temperament and character. Arch Gen Psychiatry. 1993;50:975-990.

5. Cloninger CR. Temperament and personality. Curr Opin Neurobiol. 1994;4:266-273.

6. Moresco FM, Dieci M, Vita A, et al. In vivo serotonin 5HT(2A) receptor binding and personality traits in healthy subjects: a positron emission tomography study. Neuroimage. 2002; 17:1470-1478.

7. Pecina S, Berridge KC. Opioid site in nucleus accumbens shell mediates eating and hedonic 'liking' for food: map based on microinjection Fos plumes. Brain Res. 2000;863:71-86.

8. Wester HJ, Willoch F, Tölle TR, et al. 6-O-(2-[ $\left.{ }^{18} \mathrm{~F}\right]$ fluoroethyl)-6-O-desmethyldiprenorphine $\left(\left[{ }^{18} \mathrm{~F}\right] \mathrm{DPN}\right)$ : synthesis, biologic evaluation, and comparison with $\left[{ }^{11} \mathrm{C}\right] \mathrm{DPN}$ in humans. $J$ Nucl Med. 2000;41:1279-1286.
9. Wängler B, Schneider S, Thews O, et al. Synthesis and evaluation of (S)-2-(2$\left[{ }^{18} \mathrm{~F}\right]$ fluoroethoxy)-4-([3-methyl-1-(2-piperidin-1-yl-phenyl)-butyl-carbamoyl]methyl)-benzoic acid ([$\left.{ }^{18} \mathrm{~F}\right]$-repaglinide): a promising radioligand for quantification of pancreatic $\beta$-cell mass with positron emission tomography (PET). Nucl Med Biol. 2004;31:639-647.

10. Logan J, Fowler JS, Volkow ND, Wang GJ, Ding YS, Alexoff DL. Distribution volume ratios without blood sampling from graphical analysis of PET data. J Cereb Blood Flow Metab. 1996;16:834-840.

11. Lochmann M, Buchholz HG, Siessmeier T, Schreckenberger M, Rösch F, Bartenstein P. Comparison of different quantification methods for calculating binding potential (BP) of the new unselective opiate-receptor ligand F-18 fluor-ethyl-diprenorphine. J Cereb Blood Flow Metab. 2003;23(suppl 1):669.

12. Sadzot B, Price JC, Mayberg HS, et al. Quantification of human opiate receptor concentration and affinity using high and low specific activity $\left[{ }^{11} \mathrm{C}\right]$ diprenorphine and positron emission tomography. J Cereb Blood Flow Metab. 1991;11:204-219.

13. Koepp MJ, Richardson MP, Brooks DJ, Duncan JS. Focal cortical release of endogenous opioids during reading-induced seizures. Lancet. 1998;352:952-955.

14. Friston KJ, Frith CD, Frackowiak RS, Turner R. Characterizing dynamic brain responses with fMRI: a multivariate approach. Neuroimage. 1995;2:166-172.

15. Mawlawi O, Martinez D, Slifstein M, et al. Imaging human mesolimbic dopamine transmission with positron emission tomography: I. accuracy and precision of $\mathrm{D}_{2}$ receptor parameter measurements in ventral striatum. J Cereb Blood Flow Metab. 2001;21:1034-1057.

16. Schultz W, Dayan P, Montague PR. A neural substrate of prediction and reward. Science. 1997;275:1593-1599.

17. Volpicelli JR, Watson NT, King AC, Sherman CE, O'Brien CP. Effect of naltrexone on alcohol "high" in alcoholics. Am J Psychiatry. 1995;152:613-615.

18. Suhara T, Yasuno F, Sudo $\mathrm{Y}$, et al. Dopamine $\mathrm{D}_{2}$ receptors in the insular cortex and the personality trait of novelty seeking. Neuroimage. 2001;13:891-895.

19. Kalin NH, Shelton SE, Lynn DE. Opiate systems in mother and infant primates coordinate intimate contact during reunion. Psychoneuroendocrinology. 1995; 20:735-742.

20. Zubieta JK, Smith YR, Bueller JA, et al. Regional $\mu$ opioid receptor regulation of sensory and affective dimensions of pain. Science. 2001;293:311-315.

21. Heinz A, Reimold M, Hermann D, et al. Correlation of stable elevations in striatal $\mu$-opioid receptor availability in detoxified alcoholic patients with alcohol craving: a positron emission tomography study using carbon 11-labeled carfentanil. Arch Gen Psychiatry. 2005;62:57-64.

22. Gonzales RA, Weiss F. Suppression of ethanol-reinforced behaviour by naltrexone is associated with attenuation of the ethanol-induced increase in dialysate dopamine levels in the nucleus accumbens. J Neurosci. 1998;18:10663-10671.

23. Spanagel R, Weiss F. The dopamine hypothesis of reward: past and current status. Trends Neurosci. 1999;22:521-527.

24. Gianoulakis C. Influence of the endogenous opioid system on high alcohol consumption and genetic predisposition to alcoholism. J Psychiatry Neurosci. 2001;26:304-318.

25. Willoch F, Schindler F, Wester HJ, et al. Central poststroke pain and reduced opioid receptor binding within pain processing circuitries: a $\left[{ }^{11} \mathrm{C}\right]$ diprenorphine PET study. Pain. 2004;108:213-220.

26. Spilker ME, Sprenger T, Valet $M$, et al. Quantification of $\left[{ }^{18} \mathrm{~F}\right]$ diprenorphine kinetics in the human brain with compartmental and non-compartmental modeling approaches. Neuroimage. 2004;22:1523-1533.

27. Asselin MC, Hammers A, Sethi FN, Koepp MJ, Grasby PM, Cunningham VJ. Definition of a reference region for $\mathrm{PET} /\left[{ }^{11} \mathrm{C}\right]$ diprenorphine studies [abstract]. J Cereb Blood Flow Metab. 2003;23(suppl 1):679. 